\title{
Analysis of Order Formation in Block Copolymer Thin Films \\ Using Resonant Soft X-ray Scattering
}

Justin M. Virgili, ${ }^{1,3}$ Yuefei Tao, ${ }^{2,3}$ Jeffrey B. Kortright*, ${ }^{3}$ Nitash P. Balsara*, ${ }^{1,3,4}$ and Rachel A. Segalman*1,3

${ }^{1}$ Department of Chemical Engineering and ${ }^{2}$ Department of Chemistry, University of California, Berkeley, CA 94720

${ }^{3}$ Materials Sciences and ${ }^{4}$ Energy and Environmental Technologies Divisions, Lawrence Berkeley National Laboratory, Berkeley, CA 94720

*Corresponding Author e-mails: jbkortright@,lbl.gov, nbalsara@berkeley.edu, segalman@,berkeley.edu 


\begin{abstract}
The lateral order of poly(styrene-block-isoprene) copolymer (PS-b-PI) thin films is characterized by the emerging technique of resonant soft X-ray scattering (RSOXS) at the carbon $K$ edge and compared to ordering in bulk samples of the same materials measured using hard Xray small-angle scattering. We show using theory and experiment that the loss of scattering intensity expected with a decrease in sample volume can be overcome by tuning X-rays to the $\pi^{*}$ resonance of PS or PI. Using RSOXS, we study the microphase ordering of cylinder and sphere forming PS- $b$-PI thin films and compare these results to position space data obtained by atomic force microscopy. Our ability to examine large sample areas $\left(\sim 9000 \mu \mathrm{m}^{2}\right)$ by RSOXS enables unambiguous identification of the lateral lattice structure in the thin films. In the case of the sphere forming copolymer thin film, where the spheres are hexagonally arranged, the average sphere-to-sphere spacing is between the bulk (body centered cubic) nearest neighbor and bulk unit cell spacings. In the case of the cylinder forming copolymer thin film, the cylinder-tocylinder spacing is within experimental error of that obtained in the bulk.
\end{abstract}




\section{Introduction}

Ordered block copolymer thin films ${ }^{1}$ are the subject of intense interest for nanolithographic applications ${ }^{2}$ ranging from magnetic data storage ${ }^{3,4}$ to transistors and capacitors. ${ }^{5}$ These applications require not only regularly sized, regularly spaced structures, but also control over in-plane order. As a result, a number of efforts have been made to control in plane order and orientation. ${ }^{6}$ Techniques to analyze long-range order in block copolymer thin films, however, are significantly lacking. Dynamic secondary ion mass spectrometry ${ }^{7}$ destructively detects periodic order in the direction perpendicular to the surface while neutron and X-ray reflectivity ${ }^{8-10}$ are non-destructive reciprocal space techniques capable of providing similar information, albeit with different sensitivity. Ordering in the plane of the film is generally studied via transmission electron, scanning electron, and scanning force microscopies. $^{11,12}$ Electron microscopy techniques generally require staining of an organic domain to create contrast while scanning force microscopies require careful choice of block copolymer components to create height, modulus, or other surface contrast which may be exploited by the probe tip. In fact, many block copolymer thin films contain a glassy surface brush layer that prevents imaging of the underlying pattern via scanning force techniques without a prior etching step. ${ }^{13}$ Although undersampled imaging using scanning force microscopy expands the analysis area slightly ${ }^{14,15}$ these techniques are only capable of studying areas of a few square microns at a time, yielding limited statistical information. Reciprocal space techniques have the inherent advantage of providing statistical averages over significantly larger sample sizes. The purpose of this paper is to demonstrate the utility of resonant soft X-ray scattering (RSOXS) for characterizing the in-plane order in $50 \mathrm{~nm}$ thick block copolymer films. We demonstrate that RSOXS provides an important complement to existing reciprocal space 
methods for studying lateral order in block copolymer thin films such as grazing incidence small angle X-ray scattering (GISAXS) and guided wave depolarized light scattering (GWDLS). GISAXS shows promise in allowing one to probe surface and thin film structure. ${ }^{16,17}$ However, quantitative analysis of grazing incidence scattering experiments is difficult due to the complexity in modeling GISAXS scattering data. For example, determining the in-plane structure of block copolymer thin films can be quantitative but only after scattering from both the refracted and reflected waves have been accounted for. ${ }^{16}$ GWDLS results reported thus far are qualitative in nature and restricted to films that are $1 \mu \mathrm{m}$ thick. ${ }^{18}$

In classical scattering of light, X-rays or neutrons the relationship between the transmitted and incident beam fluxes $J_{x}$ and $J_{0}$ is given by Beer's law ${ }^{19}$

$$
T=\frac{J_{x}}{J_{0}}=e^{-\mu t}
$$

where $T$ is the transmission coefficient, $\mu$ is the absorption coefficient, and $t$ the sample thickness. The optimal value of $\mu t$ for $\mathrm{X}$-ray and neutron scattering experiments is unity; i.e. $t=1 / \mu$. Samples with smaller thickness are sub-optimal due to insufficient encounters between the incident beam and scattering centers in the sample, while the incident and/or scattered beams from samples with larger thicknesses are heavily attenuated before they reach the detector. For hard $\mathrm{X}$-ray $(\sim 10,000 \mathrm{eV})$ and neutron beams, $1 / \mu \approx 1 \mathrm{~mm}$ in polymers. For soft $\mathrm{X}$-rays, $1 / \mu$ is 5 orders of magnitude smaller, i.e., $1 / \mu \approx 100 \mathrm{~nm}$. Resonant soft X-ray scattering allows us to exploit this property and study the structure of thin films. 
Studies closely related to RSOXS fall under three categories: differential anomalous small angle X-ray scattering (DAS), anomalous small angle X-ray scattering (ASAXS), and resonant hard X-ray scattering. DAS was initially applied towards structure determination in amorphous binary alloys ${ }^{20,21}$ and later in polymer electrolytes. ${ }^{22}$ ASAXS has been used to study the morphology of $\mathrm{Ni}$ neutralized ionomers ${ }^{23,24}$ and the distribution of counterions in polyelectrolyte solutions. ${ }^{25}$ Resonant hard X-ray scattering has been used to study interlayer structures in smectic liquid crystals. ${ }^{26}$ In each of these studies, X-rays were tuned to absorption edges of high atomic number $(\geq 16)$ atoms that fall in the hard X-ray regime. While similar changes in the complex scattering amplitude of selected elements is achieved in both hard and soft X-ray resonant scattering, in the hard X-ray range the intrinsic energy resolution (determined by the lifetime of the core hole in the resonant scattering process) of several electron volts is very large. The only relevant core level $(1 s)$ for organic constituents $\mathrm{C}, \mathrm{N}$, and $\mathrm{O}$ occurs in the soft $\mathrm{X}$ ray range $(280-550 \mathrm{eV})$, where much sharper intrinsic energy resolution of $\sim 0.2 \mathrm{eV}$ provides direct sensitivity to the bonds formed by these constituents.

Soft X-rays in the energy range near the carbon absorption edge (280-320 eV) have been used to investigate structure in polymer systems via Near Edge X-ray Absorption Fine Structure (NEXAFS) for contrast in conjunction with scanning transmission microscopy. ${ }^{27}$ NEXAFS microscopy is routinely used to study near surface molecular orientation and structure, but has limited resolution $(40 \mathrm{~nm})$ when studying phase separation. Soft X-ray reflectivity at the carbon absorption edge has been used to characterize the polymer-polymer and polymer-air interfaces of a poly(styrene)-poly(methylmethacrylate) bilayer. $^{28}$ RSOXS has been used to characterize porous polymer films, ${ }^{29}$ latex spheres, ${ }^{29}$ and structured latexes. ${ }^{30}$ To our knowledge, there is only one abstract in the literature in which RSOXS has been used to study block copolymer thin 
films. ${ }^{31}$ While this study clearly showed that in-plane periodicity in block copolymer thin films can be detected by RSOXS, the nature of the periodic structure could not be determined due to the lack of higher order scattering peaks. The purpose of the present paper is to determine the nature of lateral order in block copolymer thin films using higher order RSOXS peaks in a manner that is analogous the use of hard X-rays and neutrons for the study of bulk block copolymer samples. Here, we demonstrate RSOXS to be a unique tool in block copolymer structure analysis by providing radial information with the scattering vector $q$ confined strictly to the plane of the film. Further, we anticipate that since the X-ray wavelength can be tuned, much more complicated block copolymers may be studied. For instance, the ordering of an $\mathrm{ABC}$ triblock copolymer is difficult to fully understand using hard X-rays alone, but RSOXS may be used to exploit contrasts between each of the three components of the block copolymer by tuning to one or more resonance peaks of a polymer containing oxygen, nitrogen or other heteroatoms.

\section{Experimental Section}

Poly(styrene-block-isoprene) copolymers (PS-b-PI) were synthesized anionically using standard methods described in reference 32. All polymers had polydispersities less than 1.05. Polymers were stabilized with $0.7 \%$ by weight 2,6 di-tertiary-butyl-4-methyl phenol (BHT). The polymers studied are designated $\operatorname{SI}(7-79)$ and $\operatorname{SI}(4-35)$ where the numbers in parentheses refer to the number-averaged molecular weights in $\mathrm{kg} /$ mole of the poly(styrene) and poly(isoprene) blocks, respectively. The relevant polymer characteristics are summarized in Table 1.

Bulk self-assembly was characterized with small angle (hard) X-ray scattering (SAXS) performed on beamline 1-4 of the Stanford Synchrotron Radiation Laboratory (SSRL). The beamline was configured with an X-ray wavelength of $1.488 \AA$ and focused to a $0.5 \mathrm{~mm}$ 
diameter spot. Samples were prepared by annealing polymers at $180{ }^{\circ} \mathrm{C}$ (above the orderdisorder transition) under vacuum for 1 hour and then 20 hours at $110^{\circ} \mathrm{C}$ to form samples of thicknesses $1.3 \mathrm{~mm}(\mathrm{SI}(7-79))$ and $2.2 \mathrm{~mm}(\mathrm{SI}(4-35))$. Samples were held in aluminum sample cells and sealed with Kapton windows. A single quadrant of two-dimensional scattering patterns was collected on a CCD detector with a $100 \mathrm{~mm}$ diameter. The scattering patterns were radially averaged and corrected for detector null signal, dark current, and empty cell scattering. The scattering intensities were converted into absolute intensities using a poly(ethylene) standard calibrated at NIST.

PS- $b$-PI block copolymers with rubbery poly(isoprene) as the major component were chosen for this study because the modulus contrast between the microphases allowed position space imaging in tapping mode on a Digital Instruments Multimode atomic force microscope (AFM). Fifty nanometer thick films were made by spin casting $1 \%$ by weight toluene solutions of the block copolymers onto $\mathrm{Si}_{3} \mathrm{~N}_{4}$ windows for scattering experiments (SPI Supplies) and silicon substrates for AFM (University Wafer). The films were heated under vacuum for 9 hours at $120{ }^{\circ} \mathrm{C}$, followed by annealing at $95{ }^{\circ} \mathrm{C}$ for 16 hours, and finally quenched to room temperature. From earlier studies of PS- $b$-PI films it is known that PI wets the substrate and air interfaces and minor PS phases segregate away from the interfaces. ${ }^{33}$ This morphology is consistent with the films studied here. Films cast on Si wafers were initially $50 \mathrm{~nm}$ thick as confirmed by ellipsometry. Optical microscopy and AFM reveal that the surface is covered with a complete monolayer of spheres or cylinders and additional material is contained in an incomplete second layer of nanodomains (islands) on the top surface of the film. For SI(7-79), $6 \%$ of the surface is covered by a bilayer (as opposed to a monolayer) of spheres while for SI(4$35), 36 \%$ of the area is covered by a second layer of cylinders. RSOXS samples were spuncoat 
on $\mathrm{Si}_{3} \mathrm{~N}_{4}$ windows under identical conditions to those cast on $\mathrm{Si}$ wafers and should nominally be of the same thickness. Ellipsometry and AFM, however, are not possible on these small, delicate windows so the actual fractional coverage of islands is unknown. Since these substrates are substantially smaller than the $\mathrm{Si}$ wafer pieces (a few millimeters on a side as opposed to 1 $\mathrm{cm}$ ), films for RSOXS may be slightly thicker than $50 \mathrm{~nm}$. It is important to note that unlike GISAXS, RSOXS is not limited to smooth films and the presence of surface structures is not detrimental to this technique.

RSOXS experiments were conducted at beam line 6.3.2 at the Advanced Light Source (ALS), Lawrence Berkeley National Laboratory (LBNL). The PS- $b$-PI thin films were placed on a standard 2-axis diffractometer equipped with a channel electron multiplier detector that recorded the scattering intensity in photon counting mode. The beam spot size was approximately $300 \mu \mathrm{m}$ by $30 \mu \mathrm{m}$. The energy of the incident beam was tuned using a varied line spacing grating monochromator with an exit slit providing resolving power $(\mathrm{E} / \Delta \mathrm{E})$ of approximately 2000. Incident X-ray energies $(h v)$ of 265 to $325 \mathrm{eV}$, corresponding to a wavelength $(\lambda)$ range of 4.68 to $3.82 \mathrm{~nm}$ were used, and the scattering angle, $\theta$, varied from 1 to 25 degrees. We report the scattering intensity $\mathrm{I}(\mathrm{q})$ in arbitrary units after correcting for background scattering recorded from a blank $\mathrm{Si}_{3} \mathrm{~N}_{4}$ window $(\mathrm{q}=4 \pi \sin (\theta / 2) / \lambda$ is the magnitude of the scattering vector). The scattering angle was changed by simultaneously rotating the substrate by an angle $\theta / 2$ (relative to the incident beam) and the detector by an angle $\theta$. This ensures that the scattering vector always lies in the plane of the film, parallel to the substrate. Transmission-absorption spectra were collected with the X-ray beam normal to the film.

\section{Results and Discussion}




\section{Factors governing resonant scattering contrast and signal}

We begin with a discussion of the origin of X-ray scattering contrast in a binary film composed of poly(styrene) (PS) and poly(isoprene) (PI). Our treatment includes the hard X-ray spectral range where $\mathrm{X}$-ray absorption is negligible as well as the soft X-ray range where absorption is significant. The amplitude of the scattered field from each phase scales with its complex refractive index

$$
n(\lambda)=1-\delta(\lambda)-i \beta(\lambda)
$$

where $\delta$ and $\beta$ are the dispersive and absorptive contributions, respectively. Alternatively, the index can be defined as

$$
n(\lambda)=1-\frac{r_{e} \lambda^{2}}{2 \pi} \sum_{i} \rho_{i} f_{i}(\lambda)
$$

where $r_{e}$ is the electron radius, $\rho_{i}$ is the number density of atoms of type i (in PS and PI there are only 2 types of atoms: $\mathrm{C}$ and $\mathrm{H})$, and $f_{i}(\lambda)$ are the atomic scattering factors of the atoms

$$
f_{i}(\lambda)=f_{i, 1}(\lambda)+i f_{i, 2}(\lambda)
$$

where $f_{i, 1}$ and $f_{i, 2}$ are the real and imaginary contributions, respectively. Here we assume that all carbon atoms in each phase can be described by a single scattering factor for that phase $\left(f_{C, P I}\right.$ and $\left.f_{C, P S}\right)$. This simplifying assumption may not always hold in practice since different carbon 
bonds generally yield different spectral features, and significant anisotropy exists in, e.g., oriented planar molecules. These refinements will provide interesting opportunities in future studies. For now we note that $f_{C, P I}$ and $f_{C, P S}$ are assumed to represent spherically averaged values in both the phase-separated films and the neat samples whose NEXAFS spectra were used to obtain them.

The X-ray absorption length in equation 1 is given by

$$
1 / \mu=\lambda / 4 \pi \beta
$$

The scattering contrast between the PS and PI phases is proportional to $\Delta n \Delta n^{*} / \lambda^{4}$ where $\Delta n=n_{P S}-n_{P I}$ and $\Delta n^{*}$ is the complex conjugate of $\Delta n$. For a transmission geometry scattering sample with illuminated area, $A$, thickness, $t$, and transmission, $T$, the scattering intensity in the single scattering limit is

$$
I \propto\left(\frac{\Delta n \Delta n^{*}}{\lambda^{4}}\right)(A t) T=\left(\frac{\left(\beta_{P S}-\beta_{P I}\right)^{2}+\left(\delta_{P S}-\delta_{P I}\right)^{2}}{\lambda^{4}}\right)(A t) T .
$$

It is instructive to separate the contributions of the two bracketed terms in equation 6; the first gives the inherent spectral contrast that shows enhancement at the carbon $K$ edge, and the second represents the sample volume. Evaluation of the first term begins with analysis of the carbon $K$ edge region of the NEXAFS spectra of PS and PI. ${ }^{34}$ Standard methods are used to convert NEXAFS spectra to $f_{2}(\lambda){ }^{21} f_{1}(\lambda)$ is obtained using the Kramers-Kronig dispersion relation. Tabulated $f(\lambda)$ values for non-resonant species are available in references 35 and 36 . 
Substituting the atomic scattering factors into equation 3 gives $n(\lambda)$ (or equivalently $\beta(\lambda)$ and $\delta(\lambda))$ of PS and PI. The $\beta(\lambda)$ and $\delta(\lambda)$ values near the carbon $K$ edge for PS and PI thus obtained are shown in Figure 1. In both phases we find sharp resonant absorption lines at $285 \mathrm{eV}$ in $\beta$ that result from dipole transitions from $1 s$ to empty $\pi^{*}$ states (this energy is characteristic of $\mathrm{C}=\mathrm{C}$ double bonds). In contrast, the broad absorption peaks at energies above $288 \mathrm{eV}$ are predominantly from $1 s$ to $\sigma^{*}$ transitions. In our RSOXS experiment we exploit the presence of the sharp absorption lines at the $\pi^{*}$ resonance. Note that our scattering contrast (equation 6) arises from the difference $n_{\mathrm{PS}}-n_{\mathrm{PI}}$. The $\pi^{*}$ resonances in PS and PI have slightly different shapes and intensities due to differences in the carbon-carbon double bond orbital energies in aromatic PS and aliphatic PI. This difference between two sharply peaked functions can be significant even if they are of comparable magnitude and located at similar abscissa values, provided they are sharp enough. The $\pi^{*}$ resonances in Figure 1 satisfy this criterion. In contrast, the $\sigma^{*}$ peaks are much broader and thus are not useful for RSOXS in these experiments. In this case, the $\mathrm{C} \pi^{*}$ resonance was used to obtain scattering contrast, but if only one of the component phases contained a light element such as $\mathrm{N}$ or $\mathrm{O}$, then the presence and absence of resonant scattering at certain energies ( $400 \mathrm{eV}$ for $\mathrm{N}$ and $530 \mathrm{eV}$ for $\mathrm{O})$ can be the source of the scattering contrast.

In Figure 2 we show $\beta(\lambda) / \lambda^{2}$ and $\delta(\lambda) / \lambda^{2}$ of PS and PI over a wide range of energy (100 to $30,000 \mathrm{eV}$ ) in which values obtained from measured NEXAFS are spliced into tabulated values away from the carbon $K$ edge. ${ }^{35}$ It is clear that both $\beta$ and $\delta$ contributions are comparable near the carbon $K$ edge. In the hard X-ray range $(10,000 \mathrm{eV}), \beta$ is negligible compared to $\delta$. The contrast term in equation $6, \Delta n \Delta n^{*} / \lambda^{4}$, is the sum of the square of the difference between the $\beta(\lambda) / \lambda^{2}$ and $\delta(\lambda) / \lambda^{2}$ curves for PS and PI in Figure 2. We plot $\Delta n \Delta n^{*} / \lambda^{4}$ versus energy for PS 
and PI phases in the region near the carbon $K$ edge (Figure 3a) and over a wide energy range (Figure 3d). At energies above the edge (above $\sim 300 \mathrm{eV}$ ), the value of $\Delta n \Delta n^{*} / \lambda^{4}$ is remarkably constant. Near the carbon $K$ edge, however, we see an increase in $\Delta n \Delta n^{*} / \lambda^{4}$ by about two orders of magnitude. In Figures $3 \mathrm{~b}$ and $3 \mathrm{e}$ we show the energy dependence of the X-ray absorption length, $1 / \mu=\lambda / 4 \pi<\beta>$, where $<\beta>$ is the average value of $\beta$ of PS and PI. In this calculation we use the arithmetic mean of the $\beta$ values of PS and PI. For specific samples, the actual composition of the sample should be used; however, the quantitative features discussed are insensitive to the differences in $\langle\beta$. In the hard $\mathrm{X}$-ray range $1 / \mu$ approaches the $1-10 \mathrm{~cm}$ range, while near the carbon $K$ edge $1 / \mu$ is about $100 \mathrm{~nm}$. In Figures $3 \mathrm{c}$ and $3 \mathrm{f}$, we plot the scattering intensity obtained by multiplying the contributions in Figures 3a and $3 \mathrm{~d}\left(\Delta n \Delta n^{*} / \lambda^{4}\right)$ with the contributions in Figures $3 \mathrm{~b}$ and $3 \mathrm{e}(1 / \mu)$, respectively, assuming that the optimal sample thickness $t_{0}=1 / \mu$ is used to obtain the scattering. The scattering intensity at the resonance from a $100 \mathrm{~nm}$ film is only approximately a factor of 100 lower than that obtained in a standard SAXS experiment using hard X-rays and a $1 \mathrm{~mm}$ thick film in spite of the decrease in the sample volume by four orders of magnitude. It should be clear from Figure 3 that the RSOXS signal from PS- $b$-PI block copolymer thin films is facilitated by both large contrast enhancement and small penetration depth.

In Figure 4 we plot the theoretically predicted scattering intensity (line) as a function of energy for a $50 \mathrm{~nm}$ thick PS- $b$-PI film accounting for all of the three terms in equation 6 and the finite energy resolution of our instrument. The latter was taken to be a Gaussian function with a full width at half maximum (FWHM) of $0.2 \mathrm{eV}$. The symbols in Figure 4 show data obtained from $\operatorname{SI}(7-79)$ at the lowest order structural peak observed by RSOXS labeled $\mathrm{q}^{*}$ in Figure $7 \mathrm{a}$ (below). The two data sets are offset vertically for comparison, and the intensity scale is 
logarithmic. It is evident that the main features of the experimentally determined $I(h v)$ spectrum (location and height of main peak) are in general agreement with this theoretical model. Weaker features away from the predominant $\pi^{*}$ line at $284.6 \mathrm{eV}$ are in less good agreement. This is probably because of differences in spectral features of pure PS and PI used in the model calculations and microphase separated samples, due to the presence of interfaces in the latter case. Additional differences are expected due to structural interference effects that have not been included in the model. In spite of these differences, we feel that the level of agreement see in Figure 4 is sufficient to demonstrate the general validity of our theoretical description.

\section{Structural analysis of block copolymer thin films}

In Figure 5 we show a series of scattering intensity versus energy scans obtained at selected angles, using a three-dimensional $I(q, h v)$ plot from a $50 \mathrm{~nm} \mathrm{SI(7-79)}$ thin film. The $q$ value varies slightly along each scan because of the relationship $q=4 \pi \sin \theta / \lambda$. The $\pi^{*}$ resonance dominates the $I(q, h v)$ plots at all scattering angles; indeed the structural peak is not observed above and below the $\pi^{*}$ resonance energy, emphasizing the essential value of the resonant enhancement in these studies. The thick solid curve in Figure 5 shows the scattering profile at $h v=284.5 \mathrm{eV}$ and is analogous to the $I(q)$ scattering profiles observed in typical SAXS experiments in the hard X-ray regime. The primary peak at $\mathrm{q}^{*}=0.2 \mathrm{~nm}^{-1}$ indicates the presence of an in-plane periodic structure.

We first discuss results obtained from a bulk SI(7-79) sample which forms spheres packed on a body centered cubic (BCC) lattice. The bulk structure was confirmed with SAXS using hard X-rays which revealed a primary peak at $\mathrm{q}^{*}=0.223 \mathrm{~nm}^{-1}$ and higher order peaks at 0.271 and $0.312 \mathrm{~nm}^{-1}$ (Figure 6a). The bulk sphere-to-sphere spacing along the unit cell edge 
(abulk) was determined to be $39.9 \mathrm{~nm}$ from the SAXS analysis. Note that the shortest sphere-tosphere spacing in BCC is along the body diagonal and is $\sqrt{3} / 2 * a_{\text {bulk }}=34.6 \mathrm{~nm}$. A broad peak was observed at $0.567 \mathrm{~nm}^{-1}$ corresponding to a maximum in the spherical form factor $\left(\mathrm{R}_{\text {micelle }}\right)$. Following the analysis of BCC block copolymer spheres of Thomas, et al., ${ }^{37}$ we determine the radius of the PS spheres to be $9.2 \mathrm{~nm}$. Our structural characterization results are summarized in Table 1.

Microscopy studies of block copolymer thin films indicate that spheres in sphere-forming block copolymers organize on a hexagonal (HEX) lattice, although the difference between a hexagonal lattice and the distorted hexagonal lattice of the (110) plane in the BCC lattice is subtle. ${ }^{37,38}$ In Figure 7a we show an AFM micrograph of SI(7-79). The use of AFM to quantify lateral domain spacing is complicated by the fact that the instrument alternately compresses and stretches images in the slow scan direction of successive scans due to drifting of the piezo. ${ }^{39}$ Small amounts of drift are associated not only with previous changes in scan location and scan size but also with the presence of drafts and gradients in ambient temperature and are generally unavoidable. This effect can best be seen by examining the asymmetry of two-dimensional Fourier transforms of the position space data along the slow and fast scan directions. This asymmetry was corrected, in this work, by compressing or expanding the data along the slow scan axis to achieve images with symmetric 2D Fourier transforms. Both the position space image and the 2D Fourier transform shown in Figure 7a were obtained after these corrections were made. The presence of numerous spots in the Fourier transform indicates the presence of numerous grains in the scanned portion of the sample with concomitant defects. The difference between the (110) closest packed plane of a BCC structure and the close packed hexagonal lattice is only a very subtle stretching along the 100 direction of the BCC lattice. Resolving 
between these structures with position space images is thus not easy when the sample contains many defects and the raw images exhibit likely distortions as discussed above. It is thus difficult to establish the symmetry of the lattice formed by $\operatorname{SI}(7-79)$ based on the position space data in Figure 7a.

In Figure $7 \mathrm{~b}$ we show RSOXS I $(q, h v=284.5 \mathrm{eV})$ of $\operatorname{SI}(7-79)$ in a log-linear format. The sharp primary scattering peak at $\mathrm{q}^{*}=0.200 \mathrm{~nm}^{-1}$ and higher order peaks at $\sqrt{3} \mathrm{q}^{*}$ and $2 \mathrm{q}^{*}$ are clear signatures of the presence of layers of hexagonally packed spheres. The missing $\sqrt{ } 2 \mathrm{q}^{*}$ peak, which is a signature of scattering from a $\mathrm{BCC}$ lattice, demonstrates the difference between the bulk and thin film structure. The sphere-to-sphere spacing of $36.3 \mathrm{~nm}$ determined by RSOXS $\left(a_{\text {film }}\right)$ is within experimental error of the sphere spacing as measured by AFM (34.9 nm) (Table 1).

Experiments were also conducted with SI(4-35), which forms hexagonally packed cylinders in the bulk. The bulk structure was confirmed with SAXS with a primary peak observed at $\mathrm{q}^{*}=0.309 \mathrm{~nm}^{-1}$ and higher order peaks at $0.529,0.806$, and $0.908 \mathrm{~nm}^{-1}$ (Figure 6b). The bulk cylinder-to-cylinder spacing ( $\mathrm{c}_{\text {bulk }}$ ) was determined to be $23.5 \mathrm{~nm}$ by SAXS.

In Figure 8a we show an AFM micrograph of a $50 \mathrm{~nm}$ film of SI(4-35). The PS cylinders are observed to orient with their long axis parallel to a $\mathrm{SiO}_{2}$ surface based on the lower surface energy between $\mathrm{PI}$ and both the $\mathrm{SiO}_{2}$ and free surfaces present in the thin film. This observation is consistent with previous observation of PI wetting layers at both the free and $\mathrm{SiO}_{2}$ surfaces in block copolymer thin films of PS spheres in a PI matrix. ${ }^{33}$ The AFM image has been corrected for piezo drift by the previously described method to produce symmetric 2D Fourier transforms. The two regions of high intensity in the 2D Fourier transform (inset, Figure 8a) show a preferred 
orientation of many PS cylinders in the $1.5 \times 1.5 \mu \mathrm{m}$ AFM scan, indicating the presence of a large grain within the scan.

In Figure $8 \mathrm{~b}$ we show RSOXS I $(q, h v=284.5 \mathrm{eV})$ of the SI(4-35) thin film in a log-linear format. A sharp primary scattering peak is seen at $\mathrm{q}^{*}=0.261 \mathrm{~nm}^{-1}$. The second order peak at $2 \mathrm{q}^{*}$ is much stronger than the third order peak at $3 \mathrm{q}^{*}$. As discussed earlier, the films used in this study were not smooth and in fact contained a fractional coverage of a second layer of cylinders. This is one major advantage of the RSOXS technique: the presence of surface structures is not detrimental, and in fact RSOXS may be used to understand the relationship of structure in these layers. We expect the second layer to register with and orient parallel to the first layer but with second layer cylinders located in the gaps between first layer cylinders. ${ }^{40}$ Because the scattering vector is oriented strictly in the film plane for these data, the RSOXS peaks reflect the projected in-plane spacing of the 1 or 2 layer thick regions. The relevant periodicity in the monolayer regions is simply the nearest-neighbor spacing, while that in the bilayer regions is precisely half of that value. With this understanding we note that the sharp primary peak at $\mathrm{q}^{*}=0.261 \mathrm{~nm}^{-1}$ corresponds to a cylinder-to-cylinder spacing $\left(\mathrm{c}_{\text {film }}\right)$ of $24.1 \mathrm{~nm}$, which is quite close to the bulk spacing obtained by SAXS (Table 1). This $\mathrm{q}^{*}$ peak is forbidden in the bilayer regions leading to the conclusion that only the monolayer regions contribute to the $\mathrm{q}^{*}$ peak. For the bilayer regions the fundamental peak occurs at $2 q^{*}$. Furthermore, the $2 q^{*}$ peak from the bilayer regions results from the same spacing as the $\sqrt{ } 3 \mathrm{q}^{*}$ peak in the bulk sample. Possible higher harmonics of $\mathrm{q}^{*}$ from monolayer regions are allowed at $\mathrm{nq}^{*}$ for $\mathrm{n}=2,3,4, \ldots$, and for the bilayer regions at twice these values. Thus the $2 \mathrm{q}^{*}$ peak can have contributions from both mono- and bilayer regions, while the just visible feature at $3 \mathrm{q}^{*}$ results from monolayer regions only. 
For SI(4-35), the nearest-neighbor spacing between PS cylindrical domains is measured to be $3 \pm 4 \%$ larger in the thin film compared to the bulk ( $\left.\mathrm{c}_{\text {bulk }} / \mathrm{c}_{\mathrm{film}}\right)$. For SI(7-79), the nearest neighbor spacing observed in the plane of the thin film is between the two relevant distances in the BCC lattice: spacings between the 100 planes $\left(a_{\text {bulk }} / a_{\text {film }}=1.10 \pm 0.04\right)$ and the nearest neighbor spacing $\left(\sqrt{3} / 2 * a_{\text {bulk }} / a_{\text {film }}=0.95 \pm 0.04\right)$. While this appears consistent with the expected rearrangement of spheres from 2D hexagonal planes in thin films to BCC lattices in the bulk, ${ }^{37}$ a more in-depth understanding of surface reconstruction and the effect of film thickness on nanostructure are the subject of ongoing work.

\section{Conclusions}

The ability to characterize the lateral order over large areas of block copolymer thin films has been demonstrated using RSOXS. We have shown theoretically that the combination of small differences in the strong $\pi^{*}$ resonance of PS and PI and the polymers' small absorption length in the soft X-ray regime can be exploited to obtain sufficient scattering intensity to overcome the roughly four orders of magnitude decrease in sample volume in a thin film sample versus a typical SAXS sample. The energy-dependent scattering intensity measured from PS- $b$ PI films compares qualitatively with theoretically predicted scattering profiles. RSOXS characterization of block copolymer thin films should be extendable to any block copolymer system in which a resonant transition is sharply defined with respect to energy and is sufficiently spaced vis-à-vis other resonant transitions and with respect to the energy resolution limit of the monochromator. The lateral order of sphere and cylinder forming PS- $b$-PI thin films was characterized with AFM and RSOXS. Two-dimensional Fourier transforms of the real space AFM images demonstrated localized order; however, definitive assignment of the lattice type is 
complicated due to drift of the AFM piezo. In contrast, RSOXS scattering profiles revealed higher order peaks, enabling the unambiguous assignment of lattice type as well as characterization over surface areas $\sim 1000$ times larger than single AFM scans. The sphere-tosphere spacing in thin films was found to be between the bulk nearest neighbor spacing $\left(\sqrt{3} / 2 * \mathrm{a}_{\text {bulk }}\right)$ and the bulk lattice spacing $\left(\mathrm{a}_{\text {bulk }}\right)$, while the cylinder-to-cylinder spacing was found to be conserved between the bulk and thin film form within experimental error.

\section{Acknowledgements}

We gratefully acknowledge support from the Department of Energy, Office of Basic Energy Sciences, under Contract No. DE-AC03-76SF00098, support from the Plastic Electronics Program at LBNL, and support from the National Science Foundation (DMR-0514422). RSOXS experiments were performed at the ALS and SAXS experiments were performed at the SSRL. Both are national user facilities supported by the Department of Energy, Office of Basic Energy Sciences. We gratefully acknowledge Dr. John Pople for experimental assistance at the SSRL. 
Table 1: Block Copolymer Characteristics and Center-to-Center Domain Spacing

\begin{tabular}{|c|c|c|c|c|c|c|c|c|}
\hline Polymer & $\begin{array}{c}\text { Molecular } \\
\text { Weight } \\
(\mathrm{g} / \mathrm{mol})\end{array}$ & $\begin{array}{c}\text { Volume } \\
\text { Fraction } \\
\text { PS }\end{array}$ & $\begin{array}{c}\text { Domain } \\
\text { morphology }\end{array}$ & $\begin{array}{c}\underline{\underline{\text { SAXS q* }}} \\
\text { Position } \\
\left(\mathrm{nm}^{-1}\right)\end{array}$ & $\begin{array}{c}\text { SAXS Bulk } \\
\text { Spacing } \\
(\mathrm{nm})\end{array}$ & $\begin{array}{c}\underline{\text { RSOXS }} \\
\mathrm{q}^{*} \\
\text { Position } \\
\left(\mathrm{nm}^{-1}\right)\end{array}$ & $\begin{array}{c}\text { RSOXS } \\
\text { Thin Film } \\
\text { Spacing } \\
(\mathrm{nm})\end{array}$ & $\begin{array}{c}\text { AFM } \\
\text { Thin Film } \\
\text { Spacing } \\
(\mathrm{nm})\end{array}$ \\
\hline SI(4-35) & 39,200 & 0.21 & cylinders & 0.309 & $23.5 \pm 0.5$ & 0.261 & $24.1 \pm 0.2$ & $24.8 \pm 2.5$ \\
\hline SI(7-79) & 85,900 & 0.10 & spheres & 0.223 & $39.9 \pm 3.1^{+}$ & 0.200 & $36.3 \pm 0.2$ & $34.9 \pm 3.5$ \\
\hline
\end{tabular}

$+39.9 \pm 3.1 \mathrm{~nm}$ is the bulk lattice spacing $\left(\mathrm{a}_{\text {bulk }}\right)$, while the bulk nearest neighbor spacing $\left(\sqrt{3} / 2 * a_{\text {bulk }}\right)$ is $34.5 \pm 2.7 \mathrm{~nm}$ 

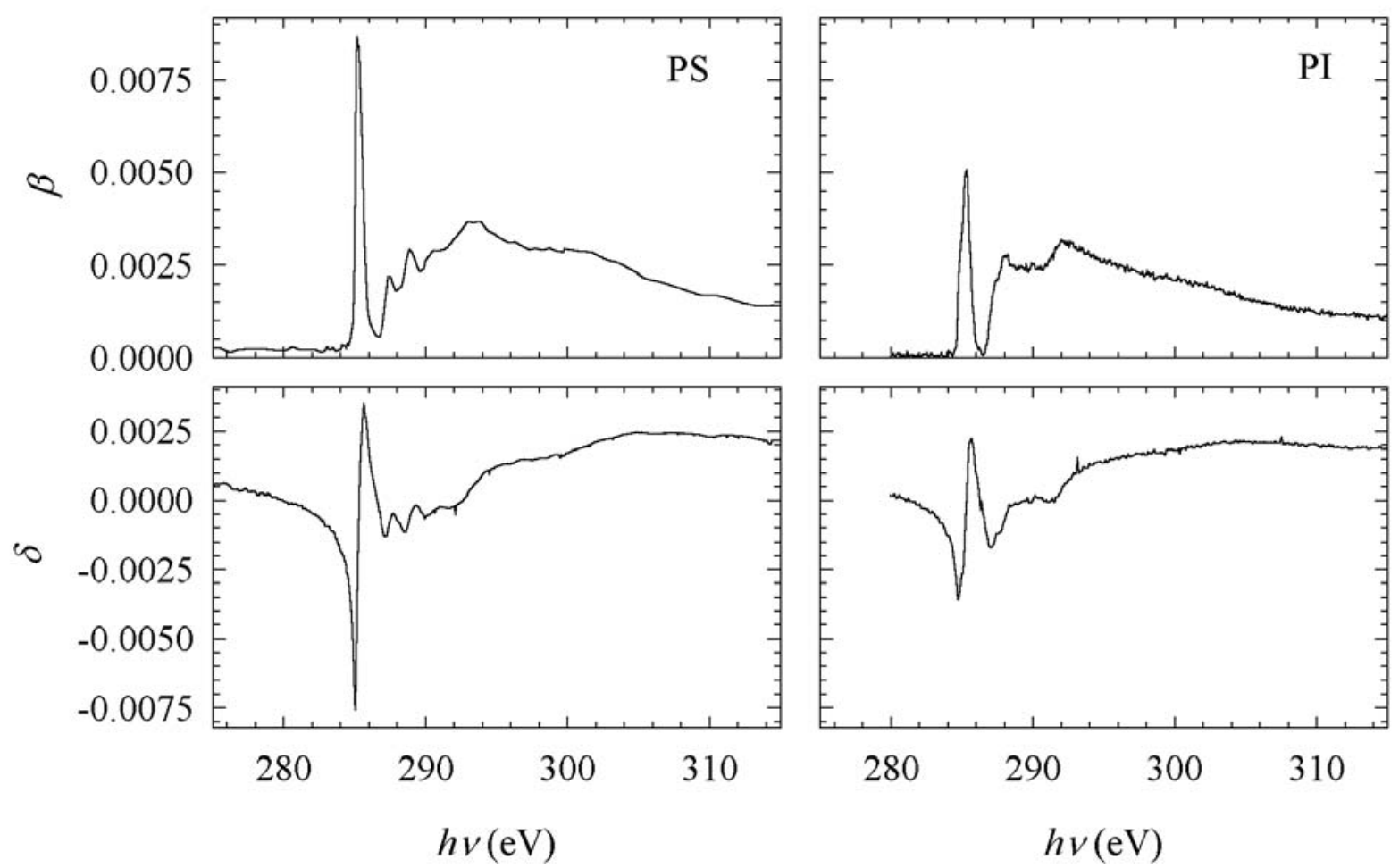

Figure 1: The real and imaginary parts of the refractive index $n=1-\delta-i \beta$ for PS and PI in the region of the carbon $K$ edge. Sharp lines near $285 \mathrm{eV}$ correspond to resonant transitions of $\mathrm{C}=\mathrm{C}$ bonds from $1 s$ to $\pi^{*}$ states. Features at higher energies result predominantly from transitions to $\sigma^{*}$ states. Data were evaluated using absorption spectra from reference 34 as described in the text. 

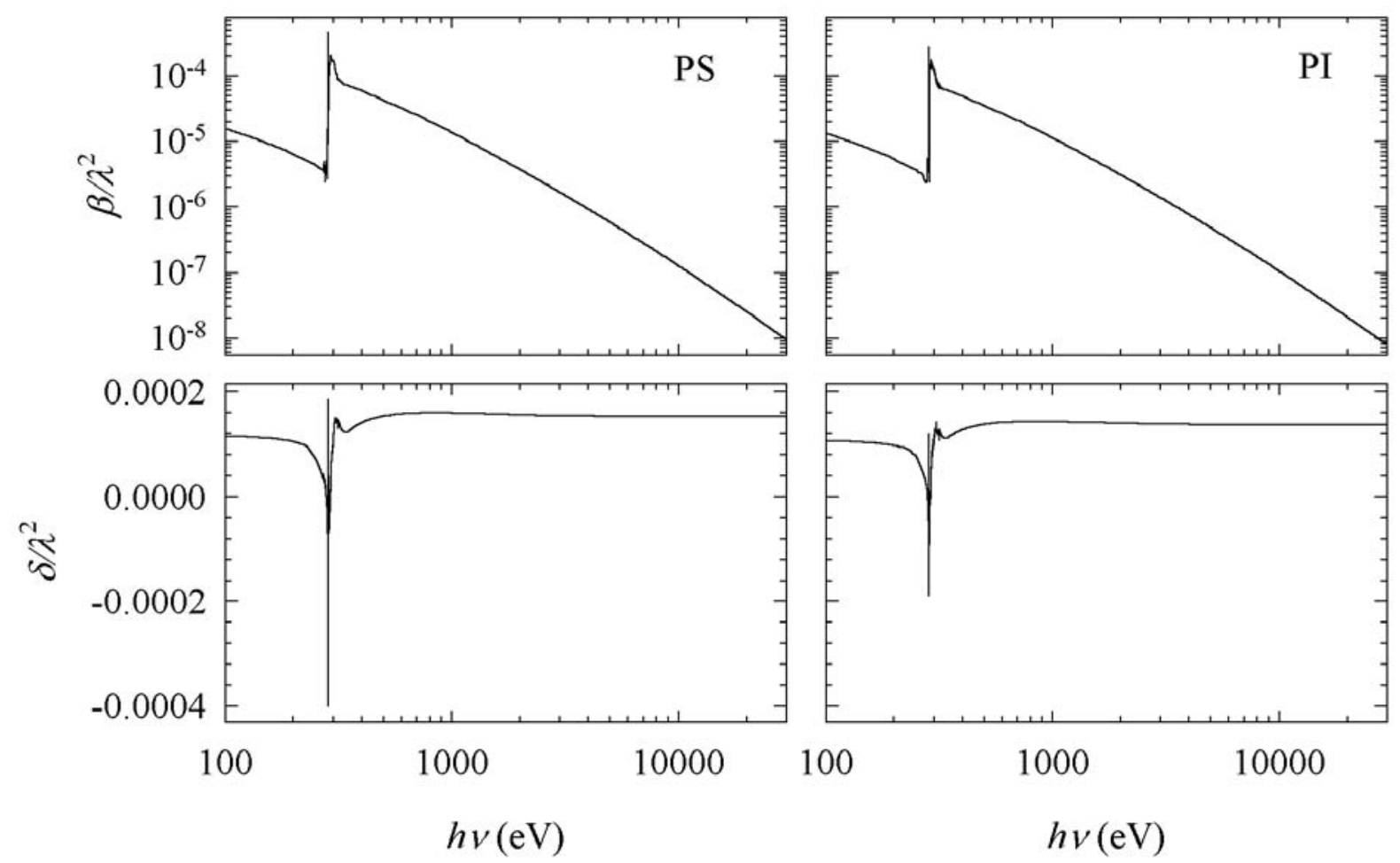

Figure 2: The imaginary (top) and real (bottom) parts of the scattering amplitude for PS and PI are plotted from below the carbon $K$ edge $(\sim 285 \mathrm{eV})$ into the hard X-ray spectral region $(\sim 10,000$ $\mathrm{eV})$. 

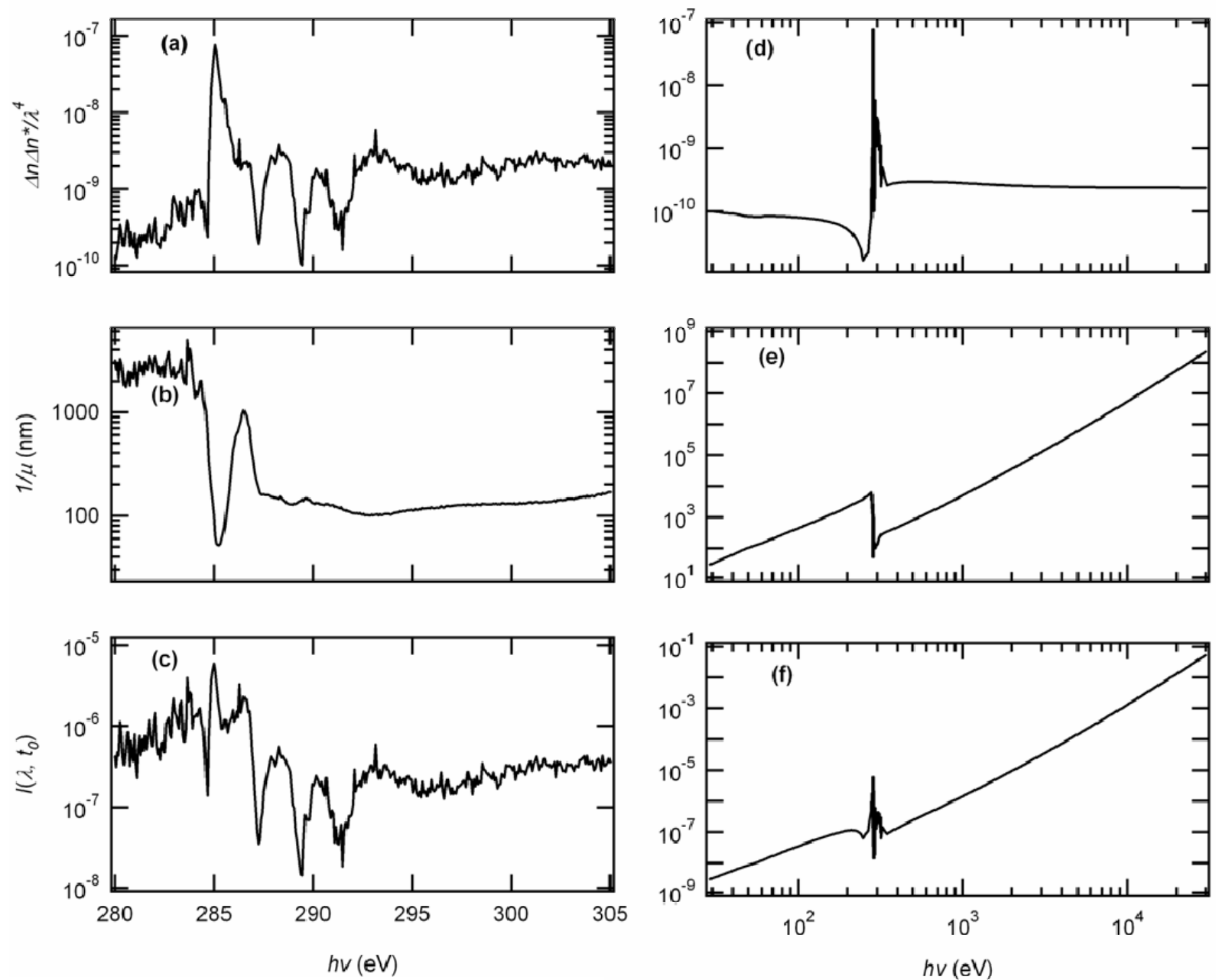

Figure 3: (a, d) The square of the scattering contrast between PS and PI vs. energy $(h v)$. (b, e) The inverse absorption length, or optimal transmission sample thickness, for a phase-separated PS-b-PI sample vs. energy. (c, f) The predicted spectral variation of the scattered intensity given by the products (a) times (b) and (d) times (e) vs. energy. The left-hand figures are enlargements of the right-hand figures in the energy range near the carbon $K$ edge. 


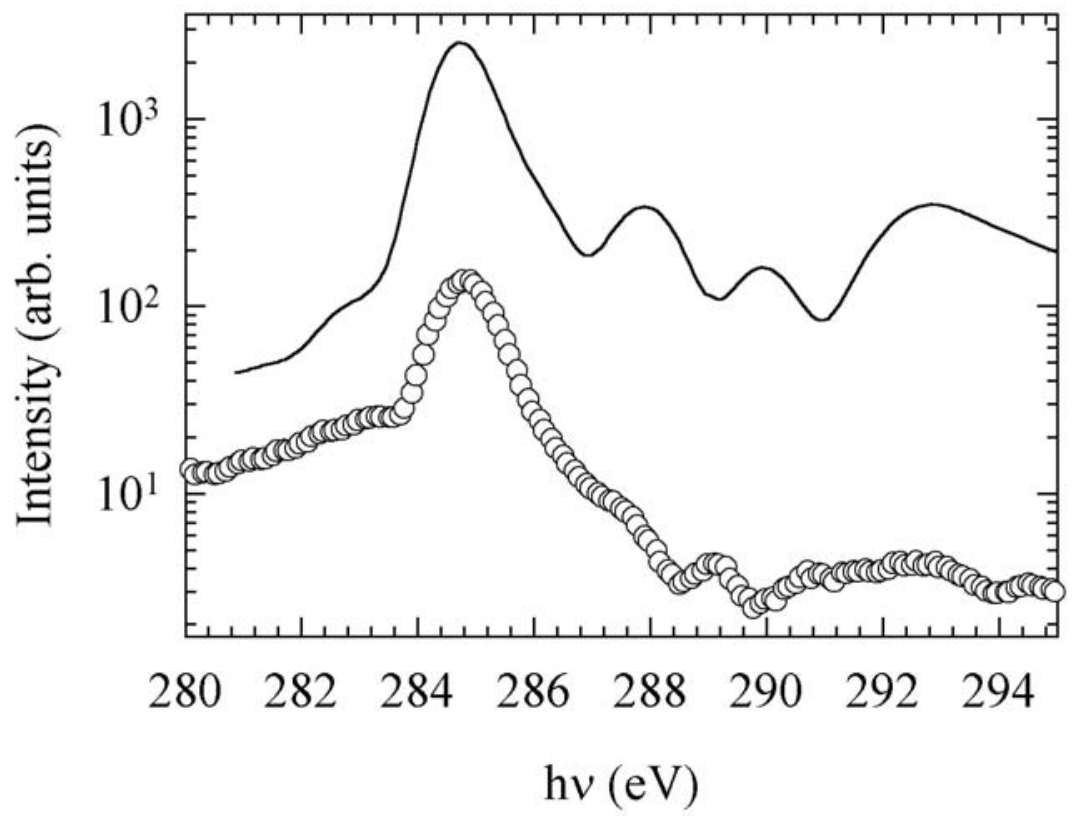

Figure 4: The predicted scattering intensity spectra from a phase-separated PS- $b$-PI film (line) compares well with a measured spectrum from a $50 \mathrm{~nm} \mathrm{SI(7-79)}$ thin film (symbols) at $\theta=3.96^{\circ}$. The model spectrum is convoluted with a Gaussian of $0.2 \mathrm{eV} \mathrm{FWHM} \mathrm{to} \mathrm{simulate} \mathrm{the} \mathrm{energy}$ resolution in the measurement, and the curves are offset vertically for clarity. 


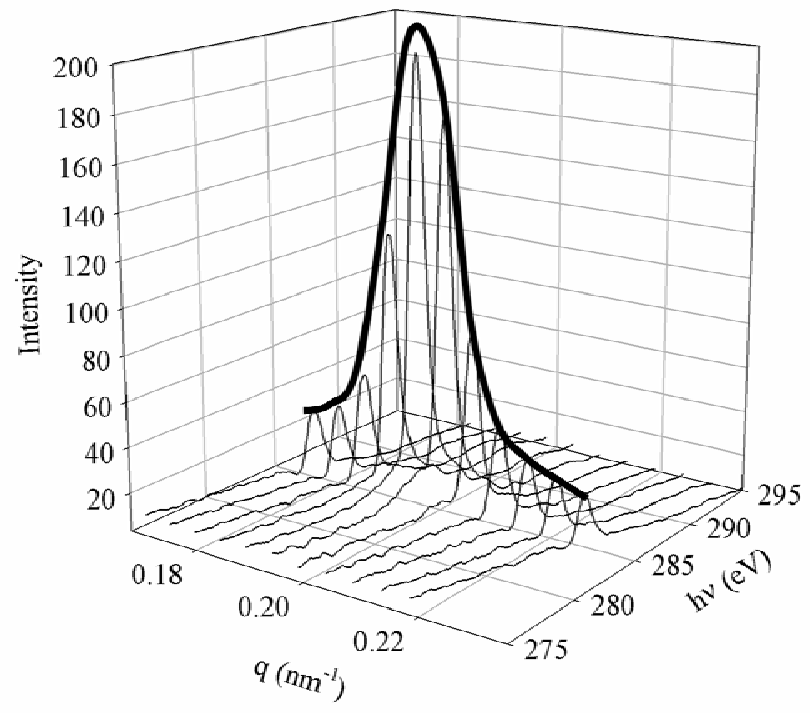

Figure 5: Scattering intensity (arbitrary units) plotted as a function of energy, $h v$, and scattering vector, $q$, near the primary scattering peak of the $50 \mathrm{~nm} \mathrm{SI(7-79)} \mathrm{thin} \mathrm{film.} \mathrm{At} \mathrm{constant} \mathrm{scattering}$ angle, maximum scattering intensities are observed at $284.77 \mathrm{eV}$. A guide to the eye (bold line) shows scattering at constant energy $(284.5 \mathrm{eV})$ in which the primary scattering peak of the block copolymer thin film is at $\sim 0.2 \mathrm{~nm}^{-1}$. 

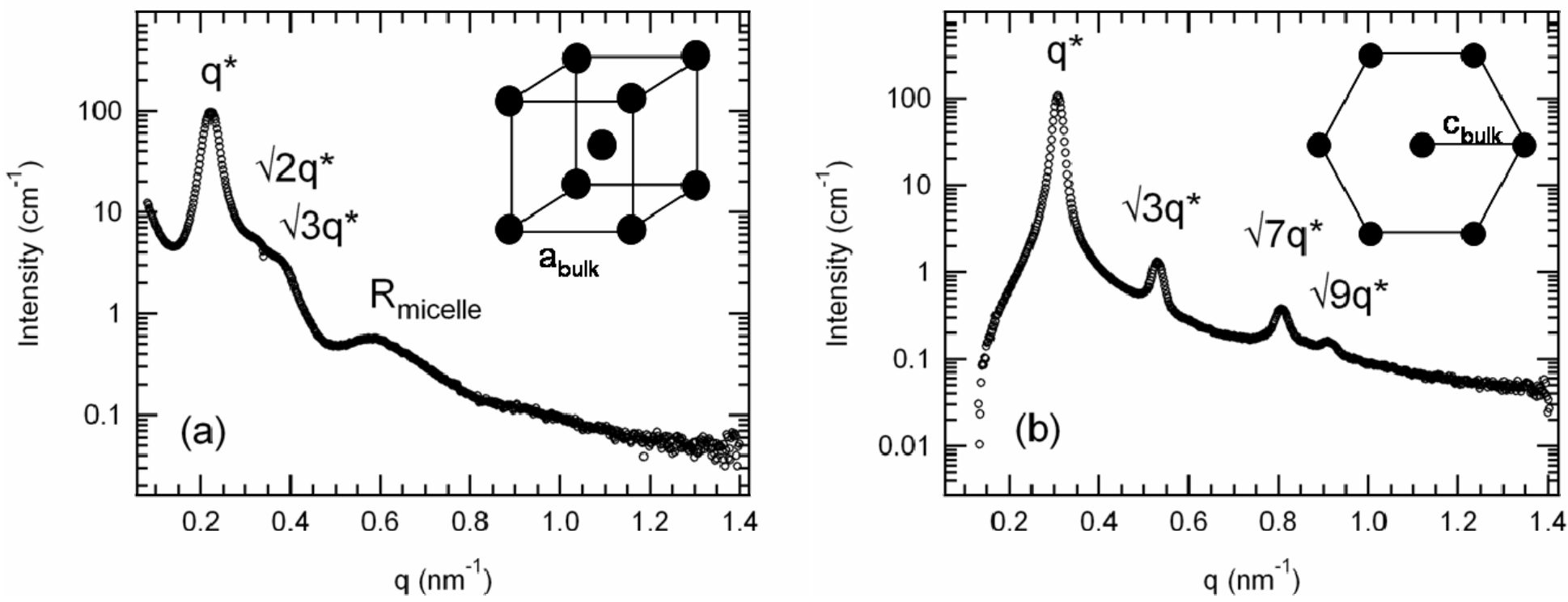

Figure 6: A) SAXS of SI(7-79) shows a primary peak at $\mathrm{q}^{*}=0.223 \mathrm{~nm}^{-1}$ and higher order peaks at $0.295 \mathrm{~nm}^{-1}$ and $0.381 \mathrm{~nm}^{-1}$ corresponding to body-centered cubic spheres. The broad peak at $\mathrm{q}$ $=0.567 \mathrm{~nm}^{-1}$ corresponds to a maximum in the scattering form factor. Our definition of the bulk lattice spacing, $\mathrm{a}_{\text {bulk}}$, is shown on the BCC lattice (inset). B) SAXS of SI(4-35) shows a primary peak at $\mathrm{q}^{*}=0.309 \mathrm{~nm}^{-1}$ and higher order peaks at $0.529,0.806$, and $0.908 \mathrm{~nm}^{-1}$ corresponding to cylinders on a hexagonal lattice. Our definition of the bulk cylinder-to-cylinder spacing, $\mathrm{c}_{\text {bulk }}$, is shown on the HEX lattice (inset). 

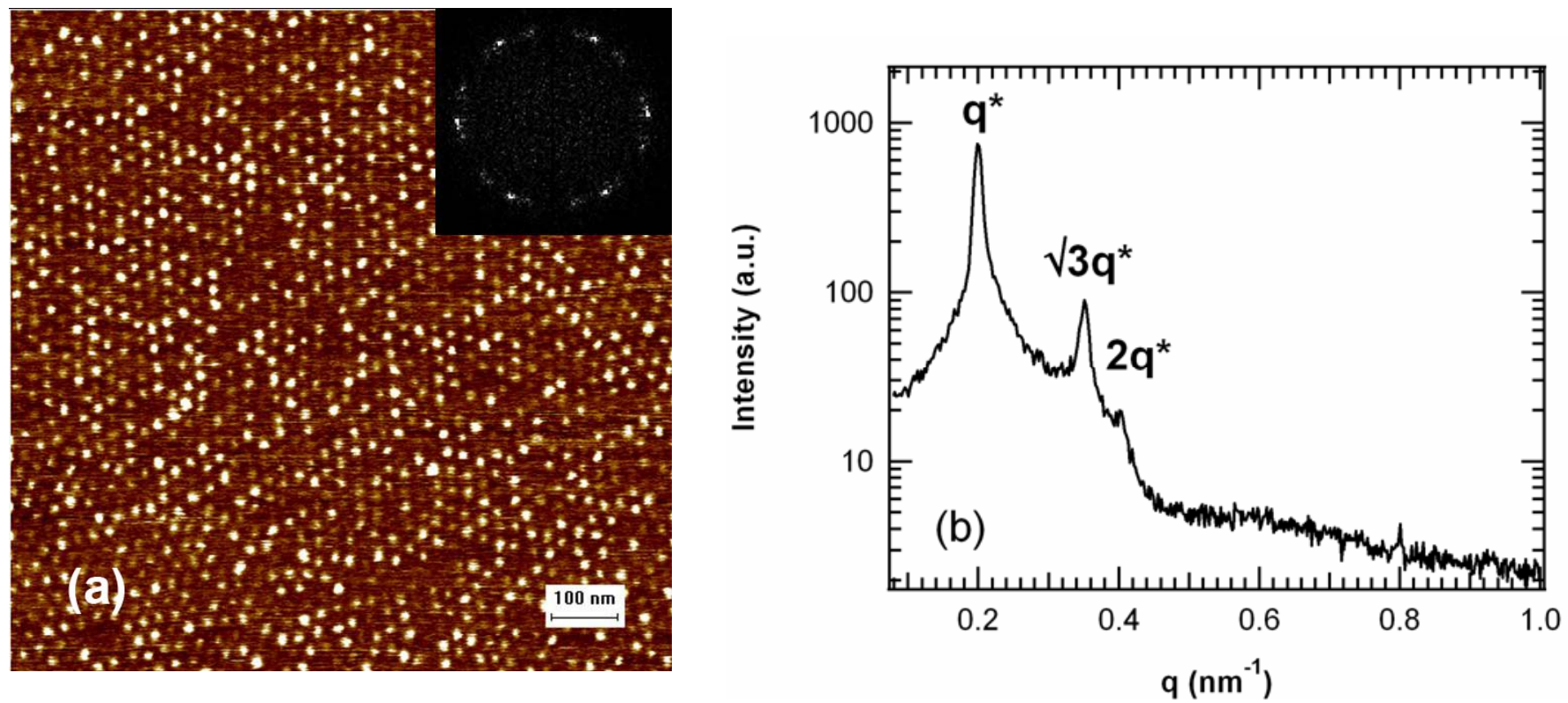

Figure 7: A) AFM phase image of a $50 \mathrm{~nm}$ sphere forming SI(7-79) thin film demonstrating polycrystallinity. B) RSOXS at the carbon edge $(284.5 \mathrm{eV})$ of a $50 \mathrm{~nm}$ thick $\mathrm{SI}(7-79)$ thin film. A primary peak at $0.200 \mathrm{~nm}^{-1}$ is observed along with higher order reflections corresponding to $\sqrt{ } 3 \mathrm{q}^{*}$ and $2 \mathrm{q}^{*}$ confirming hexagonal arrangement of PS spheres in the PI matrix. 

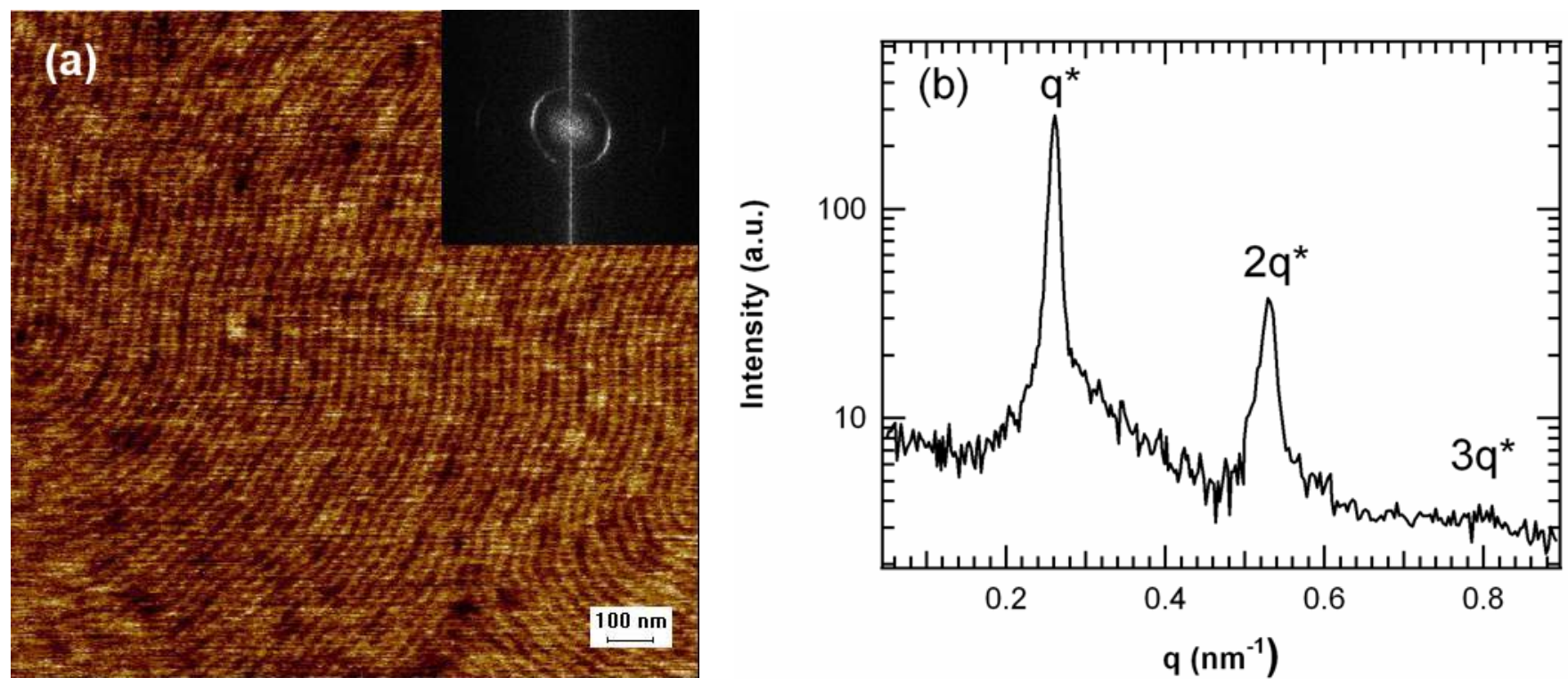

Figure 8: A) AFM phase image of a $50 \mathrm{~nm}$ cylinder forming SI(4-35) thin film. The cylinders lie parallel to the surface. B) RSOXS at the carbon edge (284.5 eV) of a $50 \mathrm{~nm} \mathrm{SI(4-35)} \mathrm{thin} \mathrm{film.}$ A primary peak at $0.261 \mathrm{~nm}^{-1}$ is observed along with $2 \mathrm{q}^{*}$ and $3 \mathrm{q}^{*}$ higher order reflections. 
For Table of Contents Use Only

Analysis of Order Formation in Block Copolymer Thin Films using Resonant Soft X-ray Scattering

Justin M. Virgili, Yuefei Tao, Jeffrey B. Kortright*, Nitash P. Balsara*, and Rachel A. Segalman*

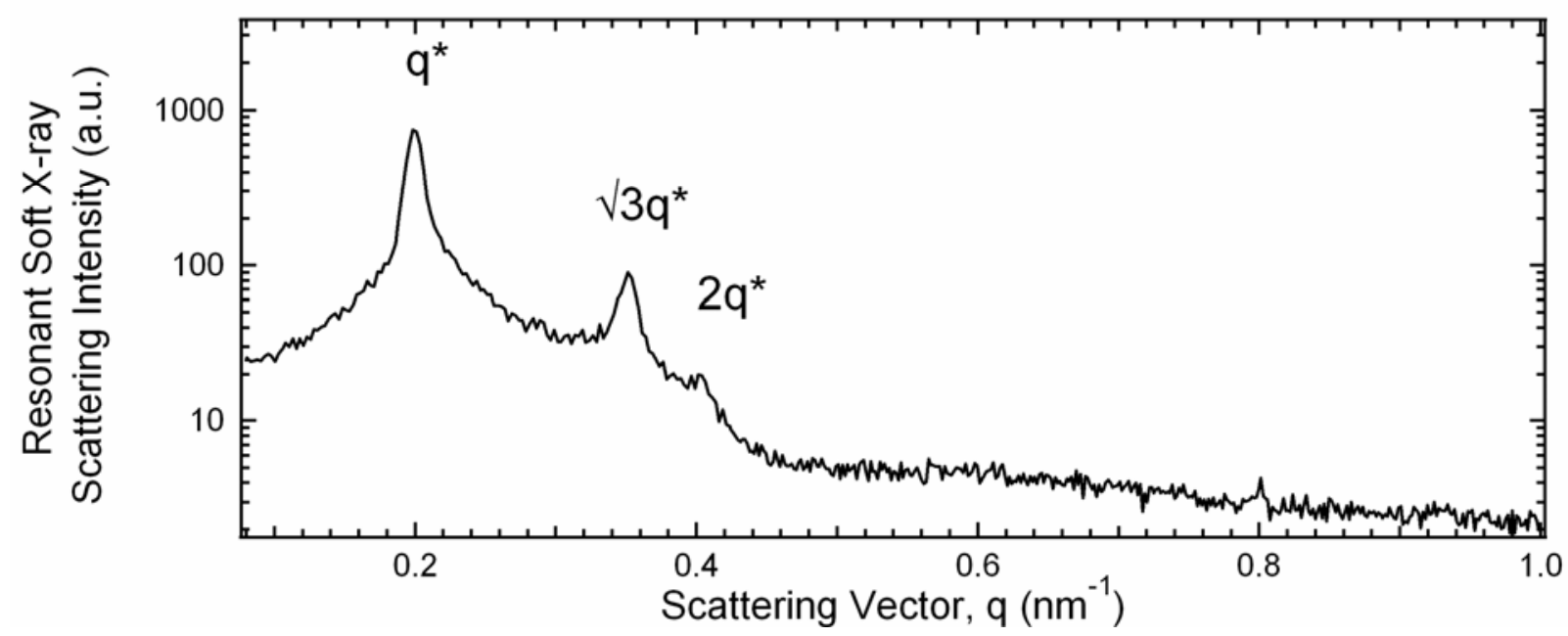




\section{References:}

1. Bates, F. S.; Fredrickson, G. H. Physics Today 1999, 52, (2), 32-38.

2. Park, M.; Harrison, C.; Chaikin, P. M.; Register, R. A.; Adamson, D. H. Science 1997, 276, (5317), 1401-1404.

3. Cheng, J. Y.; Jung, W.; Ross, C. A. Physical Review B 2004, 70, (6), 064417.

4. Cheng, J. Y.; Ross, C. A.; Thomas, E. L.; Smith, H. I.; Vancso, G. J. Applied Physics Letters 2002, 81, (19), 3657-3659.

5. Black, C. T.; Guarini, K. W.; Milkove, K. R.; Baker, S. M.; Russell, T. P.; Tuominen, M. T. Applied Physics Letters 2001, 79, (3), 409-411.

6. Segalman, R. A. Materials Science and Engineering R 2005, 48, (3), 191-226.

7. Yokoyama, H.; Kramer, E. J.; Rafailovich, M. H.; Sokolov, J.; Schwarz, S. A. Macromolecules 1998, 31, (25), 8826-8830.

8. $\quad$ Foster, M.; Stamm, M.; Reiter, G.; Huttenbach, S. Vacuum 1990, 41, (4-6), 1441-1444.

9. $\quad$ Karim, A.; Slawecki, T. M.; Kumar, S. K.; Douglas, J. F.; Satija, S. K.; Han, C. C.; Russell, T. P.; Liu, Y.; Overney, R.; Sokolov, O.; Rafailovich, M. H. Macromolecules 1998, 31, (3), 857-862.

10. Reiter, G. Macromolecules 1994, 27, (11), 3046-3052.

11. Krausch, G. Materials Science \& Engineering R-Reports 1995, 14, (1-2), 1-94.

12. Fasolka, M. J.; Mayes, A. M. Annual Review of Materials Research 2001, 31, 323-355.

13. Segalman, R. A.; Yokoyama, H.; Kramer, E. J. Advanced Materials 2001, 13, (15), 1152-1155.

14. Angelescu, D. E.; Harrison, C. K.; Trawick, M. L.; Chaikin, P. M.; Register, R. A.; Adamson, D. H. Applied Physics a-Materials Science \& Processing 2004, 78, (3), 387-392.

15. Hexemer, A.; Stein, G. E.; Kramer, E. J.; Magonov, S. Macromolecules 2005, 38, (16), 70837089.

16. Lee, B.; Park, I.; Yoon, J.; Park, S.; Kim, J.; Kim, K. W.; Chang, T.; Ree, M. Macromolecules 2005, 38, (10), 4311-4323.

17. Muller-Buschbaum, P. Analytical and Bioanalytical Chemistry 2003, 376, (1), 3-10. 
18. Garetz, B. A.; Newstein, M. C.; Wilbur, J. D.; Patel, A. J.; Durkee, D. A.; Segalman, R. A.;

Liddle, J. A.; Balsara, N. P. Macromolecules 2005, 38, (10), 4282-4288.

19. Roe, R.-J., Methods of X-Ray and Neutron Scattering in Polymer Science. Oxford University Press: New York, 2000.

20. Fuoss, P. H.; Warburton, W. K.; Bienenstock, A. Journal of Non-Crystalline Solids 1980, 35-6, (JAN-FEB), 1233-1238.

21. Kortright, J. B.; Bienenstock, A. Physical Review B 1988, 37, (6), 2979-2996.

22. Fishburn, J. R.; Barton, S. W. Macromolecules 1995, 28, (6), 1903-1911.

23. Ding, Y. S.; Hubbard, S. R.; Hodgson, K. O.; Register, R. A.; Cooper, S. L. Macromolecules 1988, 21, (6), 1698-1703.

24. Register, R. A.; Cooper, S. L. Macromolecules 1990, 23, (1), 310-317.

25. Guilleaume, B.; Blaul, J.; Ballauff, M.; Wittemann, M.; Rehahn, M.; Goerigk, G. European Physical Journal E 2002, 8, (3), 299-309.

26. Hirst, L. S.; Watson, S. J.; Gleeson, H. F.; Cluzeau, P.; Barois, P.; Pindak, R.; Pitney, J.; Cady, A.; Johnson, P. M.; Huang, C. C.; Levelut, A. M.; Srajer, G.; Pollmann, J.; Caliebe, W.; Seed, A.; Herbert, M. R.; Goodby, J. W.; Hird, M. Physical Review E 2002, 65, (4), 041705.

27. Stöhr, J., NEXAFS Spectroscopy. 2nd ed.; Springer: Santa Clara, 2003.

28. Wang, C.; Araki, T.; Ade, H. Applied Physics Letters 2005, 87, (21), 214109.

29. Mitchell, G. E.; Landes, B. G.; Lyons, J.; Kern, B. J.; Devon, M. J.; Koprinarov, I.; Gullikson, E. M.; Kortright, J. B. Applied Physics Letters 2006, 89, (4), 044101.

30. Araki, T.; Ade, H.; Stubbs, J. M.; Sundberg, D. C.; Mitchell, G. E.; Kortright, J. B.; Kilcoyne, A. L. D. Applied Physics Letters 2006, 89, (12), 124106.

31. Welch, C. F.; Hjelm, R. P.; Mang, J. T.; Wrobleski, D. A.; Orler, E. B.; Hawley, M. E.; Kortright, J. B. Abstracts of Papers of the American Chemical Society 2005, 230, U3592-U3593.

32. Lin, C. C.; Jonnalagadda, S. V.; Kesani, P. K.; Dai, H. J.; Balsara, N. P. Macromolecules 1994, 27, (26), 7769-7780. 
33. Harrison, C.; Angelescu, D. E.; Trawick, M.; Cheng, Z. D.; Huse, D. A.; Chaikin, P. M.; Vega, D. A.; Sebastian, J. M.; Register, R. A.; Adamson, D. H. Europhysics Letters 2004, 67, (5), 800-806.

34. Dhez, O.; Ade, H.; Urquhart, S. G. Journal of Electron Spectroscopy and Related Phenomena 2003, 128, (1), 85-96.

35. Henke, B. L.; Gullikson, E. M.; Davis, J. C. Atomic Data and Nuclear Data Tables 1993, 54, (2), $181-342$

36. Gullikson, E. M. http://www-cxro.lbl.gov/optical constants) 2004.

37. Thomas, E. L.; Kinning, D. J.; Alward, D. B.; Henkee, C. S. Macromolecules 1987, 20, (11), 2934-2939.

38. Henkee, C. S.; Thomas, E. L.; Fetters, L. J. Journal of Materials Science 1988, 23, (5), $1685-$ 1694.

39. Trawick, M. L.; Megens, M.; Harrison, C.; Angelescu, D. E.; Vega, D. A.; Chaikin, P. M.; Register, R. A.; Adamson, D. H. Scanning 2003, 25, (1), 25-33.

40. Harrison, C.; Park, M.; Chaikin, P. M.; Register, R. A.; Adamson, D. H.; Yao, N. Macromolecules 1998, 31, (7), 2185-2189. 